\title{
An update on organoid research
}

\author{
Organoid technologies offer unique insights into the biological processes of the tissues they mimic and are being \\ developed at a rapid pace. Here, we introduce a Collection of content from across the Nature Journals, outlining \\ recent progress and challenges in the organoid field.
}

$\mathrm{O}$ rganoids are three-dimensional tissue structures, generated from pluripotent stem cells (PSCs), which self-organise and recapitulate complex aspects of their organ counterparts, ranging from physiological processes to regeneration and disease. Since the first description of intestinal organoids in 2009 (Nature 459, 262-265; 2009), an extensive body of work has improved the methodology and expanded the range of tissues that can be studied. Their versatile nature and potential to generate human tissues endows organoids with many potential applications. They encompass research into tissue biology, development, regeneration, disease modelling, improvements in organ transplantation and drug discovery/response studies. To highlight the striking advances in this field, we are delighted to introduce our online Collection on Organoids, featuring research articles, reviews, comments and protocols published in Nature Cell Biology and in other Nature journals over the past two years (https://www. nature.com/collections/organoids).

Our selected content spans research on many different tissue types, including those of neuronal origin. Indeed, the generation of human-PSC-derived brain organoids of increasing complexity and diversity has been one of the most striking developments in this area. Researchers have established brain organoids with neuronal activity and, by varying culture conditions, have modelled more mature brain features and diverse cell types, including different neuronal and glial subtypes and photosensitive cells that are responsive to stimulation with light. Human PSCs could also be used to generate neural spheroids resembling specific brain regions that could be assembled to allow the study of regional interactions and neuronal migration. As current organoid systems lack the ability to build a vascular system, researchers recently went a step further and implanted human-derived organoids into mice to provide a functional, fully vascularized in vivo brain organoid model. The relevant papers, including a Nature Cell Biology News \& Views discussing the most important findings in four of these studies, can be found in our Collection.

Remarkable progress has also been made in recapitulating in vivo tissue features of other human organs. For instance, our Collection includes work on an organoid model of human lung development that, when transplanted into mice, mimics the formation of branching airways and early alveolar structures, and can be used to study effects of viral infection or mutations implicated in an early-onset form of pulmonary fibrosis. In another featured study, human endometrial organoids exhibited characteristics of early pregnancy when exposed to hormones. Building on the almost decade-long research on intestinal organoids, researchers have also managed to derive structures with a functional enteric nervous system that can be used to study motility disorders of the gastrointestinal tract.

The value of organoids for disease modelling and therapy development is becoming increasingly apparent. Brain organoids, for instance, have been used to model neurodegenerative and psychiatric diseases. In cancer research, tumour heterogeneity, evolution and progression have been studied using patient-derived colorectal and liver cancer organoids. Individual drug responses can be investigated, as shown in colorectal and mammary tumours. Several relevant studies included in our Collection demonstrate that tumour organoids are highly valuable preclinical models, as they permit disease modelling and exploration of treatment options in vitro and after orthotopic engraftment into mice.

To harness the potential of organoid approaches, the field has focused heavily on optimising culture conditions and defining the in vitro environments that permit stem cell expansion and differentiation into appropriate cell types. For example, synthetic hydrogels that mimic the consistency and mechanical properties of the tissue-native extracellular matrix have shown promise for efficient longterm culture of organoids. These and other technical studies, including protocols offering detailed guidance for researchers looking to use these systems in their own research, can be accessed in our Collection.

The ever-growing capacity of organoid models to closely resemble human tissues has led to increased calls for clearer guidelines and updated ethical regulations for their generation and use. The formation of advanced brain organoids in particular poses a series of important ethical questions, including the necessity to formulate metrics for 'sentient capabilities' of brain surrogates and to define boundaries in the generation of human-animal chimaeras, as highlighted by Farahany and colleagues (Nature 556, 429-432; 2018). The level of informed consent that should be obtained from human research participants is also a central question that is discussed at length by Boers and Bredenoord (Nat. Cell Biol. https://doi. org/10.1038/s41556-018-0112-5; 2018) in a specially commissioned Commentary in this issue of Nature Cell Biology. The authors argue that the progress in this field necessitates reconsideration of traditional models, which do not require consent if materials are anonymised and do not provide sufficient information to individuals who donate tissue. The authors instead advocate a model of consent for governance, which would give the donor a more prominent say in what will happen to the tissue, and additional options such as receiving on-going communication throughout the project, further participation and benefitsharing. Organoid research involving human embryonic stem cells, gametes or embryos and their clinical applications also falls under our recently updated policy (https:// go.nature.com/2rwp85k), which formalises the ethics standards of the Nature journals for such papers. Manuscripts reporting this type of work undergo review by a bioethicist and require an ethics statement outlining the institutional and ethical review process, approving the experiments and confirming that informed consent was obtained. When assessing such manuscripts editorially, we are guided by the 2016 ISSCR Guidelines for Stem Cell Research and Clinical Applications of Stem Cells (https://go.nature.com/2IrO2O0) and recommend that researchers undertaking relevant research adhere to these principles.

In presenting this Collection, we aim to capture the striking progress and potential of this methodology in basic and translational research, but also to further the discussion of ethical implications of such work. We hope our readers will find this a useful resource and thank all contributing authors, referees and journals.

Published online: 21 May 2018 https://doi.org/10.1038/s41556-018-0119-y 